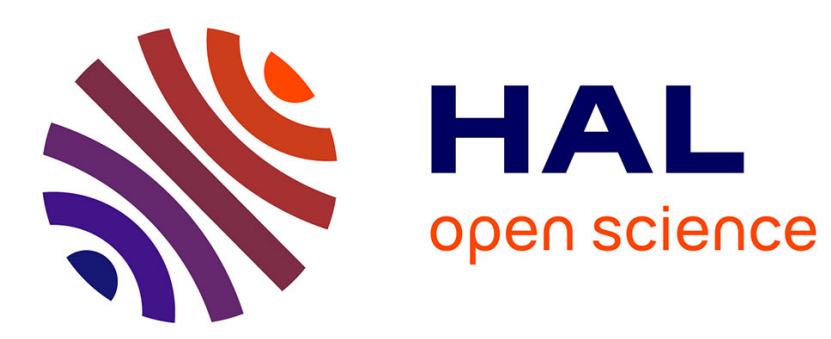

\title{
Exploiting the Graph Description of Indoor Layout for Ray Persistency Modeling in Moving Channel
}

Bernard Uguen, Nicolas Amiot, Mohamed Laaraiedh

\section{To cite this version:}

Bernard Uguen, Nicolas Amiot, Mohamed Laaraiedh. Exploiting the Graph Description of Indoor Layout for Ray Persistency Modeling in Moving Channel. European Conference on Antennas and Propagation (EUCAP 2012), Mar 2012, Prague, Czech Republic. pp.5. hal-00684269

\section{HAL Id: hal-00684269 \\ https://hal.science/hal-00684269}

Submitted on 31 Mar 2012

HAL is a multi-disciplinary open access archive for the deposit and dissemination of scientific research documents, whether they are published or not. The documents may come from teaching and research institutions in France or abroad, or from public or private research centers.
L'archive ouverte pluridisciplinaire HAL, est destinée au dépôt et à la diffusion de documents scientifiques de niveau recherche, publiés ou non, émanant des établissements d'enseignement et de recherche français ou étrangers, des laboratoires publics ou privés. 


\title{
Exploiting the Graph Description of Indoor Layout for Ray Persistency Modeling in Moving Channel
}

\author{
Bernard Uguen, Nicolas Amiot, Mohamed Laaraiedh \\ IETR UMR CNRS 6164 - University of Rennes 1 \\ Email: \{bernard.uguen, nicolas.amiot, mohamed.laaraiedh\}@univ-rennes1.fr
}

\begin{abstract}
This paper proposes a technique based on description of the layout using different graphs to obtain a ray signature which is associated with path persistency observed for small displacement of radio link termination of a mobile channel. The algorithm used to derive the signature from the layout graph description and the position of $\mathbf{T}_{x}$ and $\mathbf{R}_{x}$ is described. The mathematical relationship between signature and rays is also presented. A comparison of simulated and measured IR-UWB channel impulse response over a pedestrian trajectory is shown.
\end{abstract}

\section{INTRODUCTION}

There is today a significant research effort in localization and tracking community for measuring and modeling the features of the channel [1] for indoor human mobility. In particular, there is a growing interest for introducing channel modeling tools which respect spatial coherence of the channel w.r.t human mobility. For example semi deterministic model derived from the statistical model IEEE 802.15.4a have been proposed as e.g in [2].

The propagation channel parameters are time varying due to the motion of the extremities of the radio link or because of motion of human or objects in the propagation environment. The human motion introduces small scale and large scale variations. This paper focuses on large scale variations that remain coherent over significant distances.

Path persistency has been defined in [1] as the evolution of a particular path of the channel impulse response which exhibits a differential change of a given feature in accordance with its differential motion. In [3] the path persistency has been exploited in the context of indoor positioning systems employing high bandwidth time-of-arrival methods.

Otherwise, the graph structure has proven its relevance in describing the inner nature of the propagation channel. Stochastic propagation graphs have been introduced and developed in [4] for describing the reverberant nature of the channel and for explaining the exponential decay of the Power Delay Profile (PDP) as a function of delay.

This contribution aims at exploiting graph structure in order to determine in the channel impulse response, the part which remains persistent when the terminal is moving and can thus be exploited as a valuable observable for positioning and tracking.

All site specific tools have their own approach to describe the layout. This paper presents an approach based on a graph description of the layout which allows to capture the path persistency under the form of what is here defined as ray signature. The ray signature can remain stationary over short distances and consequently its knowledge can be exploited for accelerating the determination of rays when modeling the channel impulse response over pedestrian trajectories.

The first section introduces the proposed graph description of the layout suitable for the determination of ray signatures. The second section introduces the concept of ray signatures and describes how to derive the signature from the layout graphs. The third section provides the mathematical relationship between a ray and its signature which is used in an incremental ray tracing tool. The last section presents a comparison between channel impulse responses measured and simulated along a synthetic pedestrian trajectory in an office environment.

\section{MUltigraph DESCRIPTION OF THE LAYOUT}

The data structure of the indoor layout is described through the definition of the following graphs.

- The structure graph $\mathcal{G}_{s}$

- The visibility graph $\mathcal{G}_{v}$

- The topological graph $\mathcal{G}_{t}$

- The graph of rooms $\mathcal{G}_{r}$

The adopted multi graph description contains meta information from the layout which can be exploited for both incremental identification of rays and simulation of indoor pedestrian mobility.

\section{A. The structure graph $\mathcal{G}_{s}$}

The first graph describing the layout is $\mathcal{G}_{s}\left(\mathcal{V}_{s}, \mathcal{E}_{s}\right)$. $\mathcal{V}_{s}$ is the set of nodes and $\mathcal{E}_{s}$ is the set of edges. This is illustrated in figure 1 for 4 vertices and 4 walls room. In this example:

$$
\begin{gathered}
\mathcal{V}_{s}=\{-1,-2,-3,-4,1,2,3,4\} \\
\operatorname{card}\left(\mathcal{V}_{s}\right)=8
\end{gathered}
$$

Vertices can be associated with a diffraction interaction whereas the edge (positive index) are associated either with transmission or diffraction.

- A positive node (wall) is necessarily connected to a negative node (vertex)

- For a vertex to be a potential diffracting node, its degree has to be lower or equal to 2

Each node index allows to retrieve the associated constitutive properties of the wall. 
Figure 2 shows the $\mathcal{G}_{s}$ graph of an indoor environment. Only edges are displayed, black lines are associated with doors and windows.

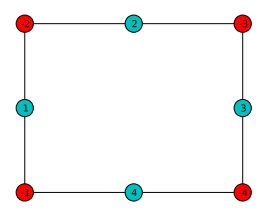

Fig. 1. $\mathcal{G}_{s}$ representation of a 4 walls layout (nodes in red: vertices, nodes in blue: walls)

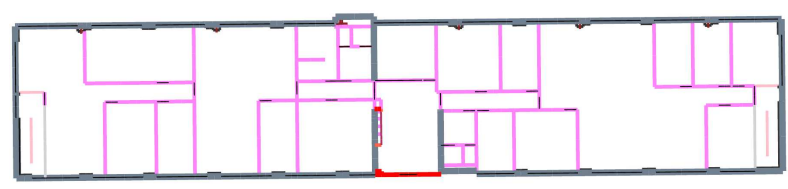

Fig. 2. The structure graph $\mathcal{G}_{s}$

\section{B. The visibility graph $\mathcal{G}_{v}$}

The second defined graph of the layout is the visibility graph $\mathcal{G}_{v}$. Nodes of this graph are the same as those of $\mathcal{G}_{s}$. Only edges are defined differently from $\mathcal{G}_{s}$. Two nodes are connected if they share an "optical" visibility relationship. A wall $(i d>0)$ can be connected to a vertex $(i d<0)$. Optical visibility means that the electromagnetic energy can flow from one node to another. The $\mathcal{G}_{v}$ graph structure is sparse and well adapted to describe visibility relationships between layout and edges.

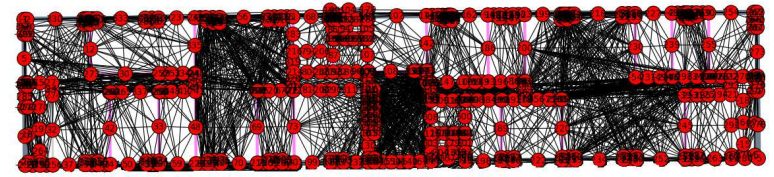

Fig. 3. Visibility graph $\mathcal{G}_{v}$

\section{The topological (cycles) graph $\mathcal{G}_{t}$}

The third defined graph of the Layout is the topological graph $\mathcal{G}_{t}$. It results from the determination of all cycles of the graph $\mathcal{G}_{s}$. $\mathcal{G}_{t}$ contains a valuable topological information. Each cycle contains various attributes as for example the set of nodes and edges of the cycle. This information is exploited once the position of $\mathbf{T}_{x}$ or $\mathbf{R}_{x}$ is known in order to connect directly leaves of $\mathcal{G}_{v}$ which are associated with a given cycle. This data organization accelerates search of walls and vertices to connect with. Graph $\mathcal{G}_{t}$ is shown in Figure 4.

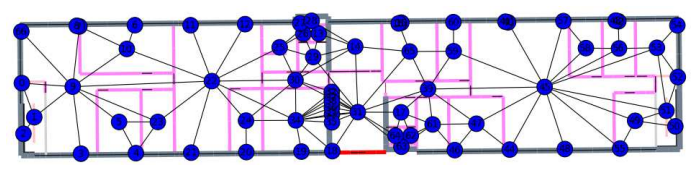

Fig. 4. Topological (cycles) graph $\mathcal{G}_{t}$

\section{The graph of rooms $\mathcal{G}_{r}$}

The fourth defined graph is the graph of rooms $\mathcal{G}_{r}$. It is the subgraph of $\mathcal{G}_{t}$ retaining only cycles which possess at least one door. An example of a graph of rooms is shown in Figure 5. This graph is used for fast extraction of the list of edges which belongs to a given room. This graph can also been used to build a state machine for inter-room mobility of pedestrian agents.

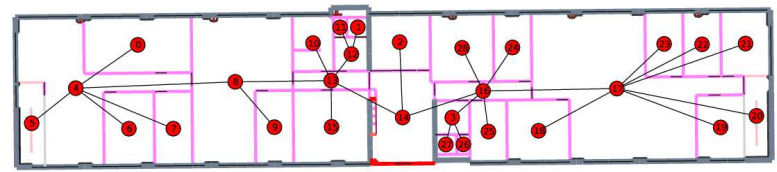

Fig. 5. Graph of rooms $\mathcal{G}_{r}$

\section{DEFINING A RAY SIGNATURE}

\section{A. Definition}

Let first define a ray with $N_{i}$ interactions as an ordered list of $N_{i}+2$ points starting at $\mathbf{t}_{x}$ and ending at $\mathbf{r}_{x}$.

$$
\mathcal{R}=\left\{\mathbf{t}_{\mathbf{x}}, \mathbf{p}_{0}, \cdots, \mathbf{p}_{N_{i}-1}, \mathbf{r}_{x}\right\}=\left\{\mathbf{t}_{x}, \mathcal{P}, \mathbf{r}_{x}\right\}
$$

where $\mathbf{t}_{x}$ and $\mathbf{r}_{x}$ are points associated with transmitter and receiver and the ordered list of $N_{i}$ interaction points is $\mathcal{P}=\left\{\mathbf{p}_{0}, \cdots, \mathbf{p}_{N_{i}-1}\right\}$. The signature of $\mathcal{R}$ is defined as the sequence of structure ids of $\mathcal{P}$. The structure id of a point is simply the identification number either of the diffraction point $(i d<0)$ ot the segment number $(i d>0)$, this is directly a node number from graph $\mathcal{G}_{s}$ or $\mathcal{G}_{v}$ defined previously. $\operatorname{Id}(\mathbf{p})$ is a function which returns the layout index number of $\mathbf{p}$.

$$
\mathcal{S}(\mathcal{R})=\mathcal{S}(\mathcal{P})=\left\{\operatorname{Id}\left(\mathbf{p}_{0}\right), \cdots, \operatorname{Id}\left(\mathbf{p}_{N_{i}-1}\right)\right\}
$$

Conventionally, the signature of the LOS path is the empty set, $\mathcal{S}\left(\left\{\mathbf{t}_{x}, \mathbf{r}_{x}\right\}\right)=\emptyset$. Notice that when at least one ending point of the radio link is moving, all the points of $\mathcal{P}$ are moving correspondingly. This is not the case of the signature $\mathcal{S}$ which can remain stationary in the vicinity region of the moving point.

\section{B. Algorithm to Determine Signature from $\mathcal{G}_{v}$}

In a first step the algorithm seeks for the nodes $\mathcal{V}_{t x}$ of $\mathcal{G}_{s}$ which are visible from $\mathbf{T}_{x}$, and the set of nodes $\mathcal{V}_{r x}$ of $\mathcal{G}_{s}$ which are visible both from $\mathbf{R}_{x}$. This is a fast operation if the information of the room numbers where are lying $\mathbf{T}_{x}$ and $\mathbf{R}_{x}$ are maintained available along the simulated trajectory. The second step consists in exploiting the 2 subsets $\mathcal{V}_{t x}$ and $\mathcal{V}_{r x}$ for 

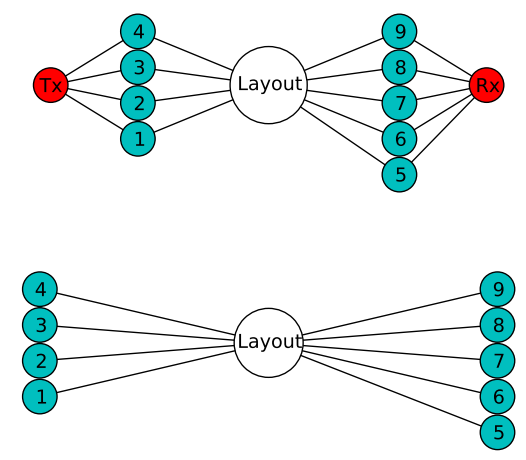

Fig. 6. Principle of signature determination, nodes subset $\mathcal{V}_{t x}$ (left) and $\mathcal{V}_{r x}$ (right)

determining $N_{t} \times N_{r}$ first order signatures where $\operatorname{card}\left(\mathcal{V}_{t x}\right)=$ $N_{t}$ and $\operatorname{card}\left(\mathcal{V}_{r x}\right)=N_{r}$. The algorithm exploits the Dijkstra algorithm to determine the shortest path between 2 nodes in the graph $\mathcal{G}_{v}$. If a keyhole is detected, i.e a central node which is present in many discovered signatures, it is possible to remove this node and to iterate the algorithm in order to get new signatures which explore less direct paths. In scenarios where one extremity is moving continuously along a trajectory the set of signature $\mathcal{L}$ calculated at one point can be exploited for determining rays in its vicinity. $\mathcal{L}$ can be incrementally updated. Figure 6 and Algorithm 1 describe the principle of rays signatures determination.

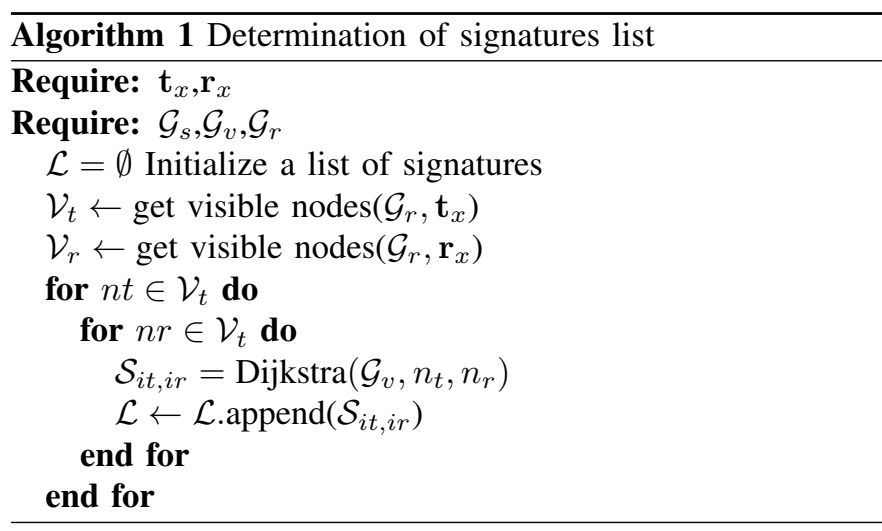

\section{Ray Determination From Ray Signature}

The determination of a ray from its signature is a two steps process. The fist step consists in calculating a set of intermediate points starting from the transmitter. Once calculated those intermediate points can be used for any receiver position with the same signature. The signature can be either valid or not valid for a given receiver depending on its coordinates.

\section{A. Determination of Image points}

The transmitter is defined as

$$
\mathbf{t}_{x}=\left[x_{t}, y_{t}\right]^{T}
$$

We consider a signature of length $N$ which can contain three types of interaction: diffraction (D), reflection (R), transmission $(\mathrm{T})$. From this signature, 2 ordered lists of points are retrieved.

$$
\begin{gathered}
\mathbf{p}_{a}=\left[\mathbf{a}_{0}, \ldots, \mathbf{a}_{N-1}\right] \\
\mathbf{p}_{b}=\left[\mathbf{b}_{0}, \ldots, \mathbf{b}_{N-1}\right] \\
\mathbf{a}_{k}=\left[x_{A k}, y_{A k}\right]^{T}, \mathbf{b}_{k}=\left[x_{B k}, y_{B k}\right]^{T}
\end{gathered}
$$

In the case of a diffraction the point appears identical in each list otherwise $\mathbf{p}_{a}$ and $\mathbf{p}_{b}$ contains respectively tail points and head points of the signature segments.

The sequence of intermediate points can be determined from the knowledge of the transmitter point, the sequence of segments and also importantly the type of interaction $(R|T| D)$. This sequence of intermediate points is:

$$
\mathbf{m}=\left[\mathbf{m}_{0}, \ldots, \mathbf{m}_{N-1}\right]
$$

with:

$$
\mathbf{m}_{k}=\left[x_{k}, y_{k}\right]^{T}
$$

Depending on the nature of the interaction the intermediate point is either the image of the current point w.r.t the segment (Reflection), the current point itself (Transmission), the diffraction (interaction) point itself (Diffraction).

$\mathbf{m}_{0}$ depends on the type of the first interaction as:

- (R): $\mathbf{m}_{0}=\mathbf{S}_{0} \mathbf{t}_{x}+\mathbf{v}_{0}$

- (T): $\mathbf{m}_{0}=\mathbf{t}_{x}$

- (D): $\mathbf{m}_{0}=\mathbf{a}_{0}$

and similarly, $\mathbf{m}_{k}$ depends on the type of the $k^{t h}$ interaction:

- (R): $\mathbf{m}_{k}=\mathbf{S}_{k} \mathbf{m}_{k-1}+\mathbf{v}_{k}$

- (T): $\mathbf{m}_{k}=\mathbf{m}_{k-1}$

- (D): $\mathbf{m}_{k}=\mathbf{a}_{k}$

where $\mathbf{S}_{k}$ (reflection matrix) and $\mathbf{v}_{k}$ are defined as:

$$
\mathbf{S}_{k}=\left[\begin{array}{cc}
a_{k} & -b_{k} \\
-b_{k} & -a_{k}
\end{array}\right]
$$

the translation vector is defined as

$$
\mathbf{v}_{k}=\left[\begin{array}{l}
c_{k} \\
b_{k}
\end{array}\right]
$$

with

$$
\begin{array}{cc}
a_{k}= & \frac{\left(x_{A k}-x_{B k}\right)^{2}-\left(y_{A k}-y_{B k}\right)^{2}}{\left(x_{A k}-x_{B k}\right)^{2}+\left(y_{A k}-y_{B k}\right)^{2}} \\
b_{k}= & \frac{2\left(x_{B k}-x_{A k}\right)\left(y_{A k}-y_{B k}\right)}{\left(x_{A k}-x_{B k}\right)^{2}+\left(y_{A k}-y_{B k}\right)^{2}} \\
c_{k}= & 2 \frac{x_{A k}\left(y_{A k}-y_{B k}\right)^{2}+y_{A k}\left(x_{B k}-x_{A k}\right)\left(y_{A k}-y_{B k}\right)}{\left(x_{A k}-x_{B k}\right)^{2}+\left(y_{A k}-y_{B k}\right)^{2}} \\
d_{k}= & 2 \frac{x_{A k}\left(y_{A k}-y_{B k}\right)\left(x_{B k}-x_{A k}\right)+y_{A k}\left(x_{B k}-x_{A k}\right)^{2}}{\left(x_{A k}-x_{B k}\right)^{2}+\left(y_{A k}-y_{B k}\right)^{2}}
\end{array}
$$

The coordinates of intermediates points are obtained by solving

$$
\mathbf{A m}=\mathbf{y}
$$




$$
\begin{aligned}
& \mathbf{A}=\left[\begin{array}{cccc}
\mathbf{I}_{2} & & & \\
\left\{-\mathbf{S}_{1}\left|\mathbf{O}_{2}\right| \mathbf{O}_{2}\right\} & \mathbf{I}_{2} & & \\
& \ddots & \ddots & \\
& & \left\{-\mathbf{S}_{N-1}\left|\mathbf{O}_{2}\right| \mathbf{O}_{2}\right\} & \mathbf{I}_{2}
\end{array}\right] \\
& \mathbf{y}=\left[\begin{array}{c}
\left.\left\{\mathbf{S}_{0} \mathbf{t}_{x}+\mathbf{v}_{\mathbf{0}} \mid \mathbf{t}_{x}\right\} \mid \mathbf{a}_{0}\right\} \\
\left\{\mathbf{v}_{\mathbf{1}}\left|\mathbf{z}_{2}\right| \mathbf{d}_{1}\right\} \\
\vdots \\
\left\{\mathbf{v}_{\mathbf{N}-\mathbf{1}}\left|\mathbf{z}_{2}\right| \mathbf{d}_{N-1}\right\}
\end{array}\right]
\end{aligned}
$$

where $\mathbf{I}_{2}$ and $\mathbf{O}_{2}$ are respectively identity matrix and zero matrix of dimension $2, \mathbf{z}_{2}$ is $2 \times 1$ zero column vector. The notation $\{R|T| D\}$ stands for the possible matrix entries depending on the interaction type.

\section{B. Determination of interaction points}

The receiver is given by:

$$
\mathbf{r}_{\mathbf{x}}=\left[x_{r}, y_{r}\right]^{T}
$$

Interaction points are points which belong to the layout segments. They are obtained from the intersection between the segments indicated by the signature and the segments joining successively the previously obtained intermediate points $\mathbf{m}$. The unknown interaction points are

$$
\mathbf{p}_{k}=\left[x_{k}^{\prime}, y_{k}^{\prime}\right]^{T}
$$

By introducing a parameterisation on both the unknown $4 \times$ 1 column vector is:

$$
\mathbf{x}_{k}^{\prime}=\left[\mathbf{p}_{k}^{T}, \Gamma_{k}^{T}\right]^{T}
$$

with

$$
\boldsymbol{\Gamma}_{k}=\left[\alpha_{k}, \beta_{k}\right]^{T}
$$

Starting from the receiver and joining the last intermediate point $\mathbf{m}_{N-1}$

$$
\begin{array}{r}
\mathbf{p}_{0}+\alpha_{0}\left(\mathbf{r}_{\mathbf{x}}-\mathbf{m}_{N-1}\right)=\mathbf{r}_{\mathbf{x}} \\
\mathbf{p}_{0}+\beta_{0}\left(\mathbf{a}_{N-1}-\mathbf{b}_{N-1}\right)=\mathbf{a}_{N-1}
\end{array}
$$

We solve successively the following linear systems

$$
\mathbf{T}_{0} \mathbf{x}_{0}^{\prime}=\mathbf{y}_{0}^{\prime}
$$

with:

and

$$
\mathbf{T}_{0}=\left[\begin{array}{ccc}
\mathbf{I}_{2} & \mathbf{r}_{\mathbf{x}}-\mathbf{m}_{N-1} & \mathbf{0}_{2 \times 1} \\
\mathbf{I}_{2} & \mathbf{0}_{2 \times 1} & \mathbf{a}_{N-1}-\mathbf{b}_{N-1}
\end{array}\right]
$$

$$
\mathbf{y}_{0}^{\prime}=\left[\mathbf{r}_{\mathbf{x}}^{T}, \mathbf{a}_{N-1}^{T}\right]^{T}
$$

For determination of interaction point $\mathbf{p}_{k}$ it proceeds identically taking advantage of the knowledge of point $\mathbf{p}_{k-1}$ :

$$
\begin{array}{r}
\mathbf{p}_{k}+\alpha_{k}\left(\mathbf{p}_{k-1}-\mathbf{m}_{N-(k+1)}\right)=\mathbf{p}_{k-1} \\
\mathbf{p}_{k}+\beta_{k}\left(\mathbf{a}_{N-(k+1)}-\mathbf{b}_{N-(k+1)}\right)=\mathbf{a}_{N-(k+1)}
\end{array}
$$

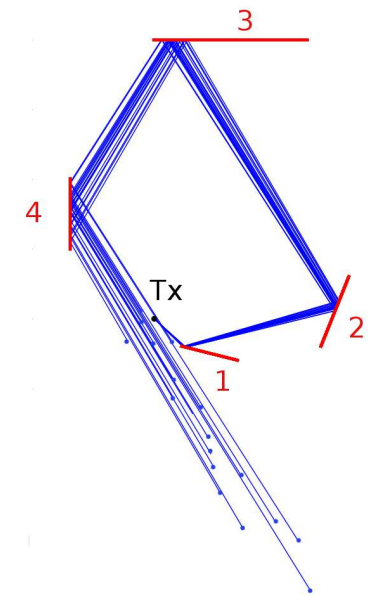

Fig. 7. Rays in blue share the same signature

This takes the forme of the linear equation:

$$
\mathbf{T}_{k} \mathbf{x}_{k}^{\prime}=\mathbf{y}_{k}^{\prime}
$$

with

$$
\mathbf{T}_{k}=\left[\begin{array}{ccc}
\mathbf{I}_{2} & \mathbf{p}_{k-1}-\mathbf{m}_{N-(k+1)} & \mathbf{0}_{2 \times 1} \\
\mathbf{I}_{2} & \mathbf{0}_{2 \times 1} & \mathbf{a}_{N-(k+1)}-\mathbf{b}_{N-(k+1)}
\end{array}\right]
$$

and

$$
\mathbf{y}_{k}^{\prime}=\left[\mathbf{p}_{k-1}^{T}, \mathbf{a}_{N-(k+1)}^{T}\right]^{T}
$$

The parameterization of the signature segment $\beta_{k}$ plays a very important role, if $0<\beta_{k}<1$ the interaction point $\mathbf{p}_{k}$ is valid and the calculation can proceed otherwise the interaction point is out of the segment and the signature is rejected for the current receiver coordinates.

As an example Figure 7 shows a set of rays (in blue) which are all sharing the same signature, which contains 4 reflections. $\mathbf{T}_{x}$ is static and is placed above-left the first segment.

The signature for all blue rays is $\mathcal{S}=\{1,2,3,4\}$. The figure has been produced by drawing randomly $\mathbf{R}_{x}$ in the plane. There exists a validity region for $\mathbf{R}_{x}$ for a ray being associated with the signature. It means that if one termination of the signature is static (e.g. an access point), either $\mathbf{T}_{x}$ or $\mathbf{R}_{x}$, one can associate the couple (access point, signature) to a validity region where the signature is associated to valid rays.

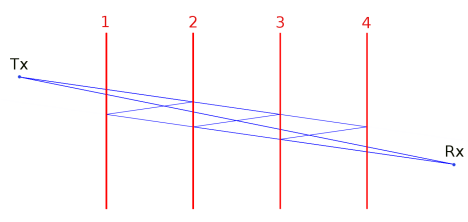

Fig. 8. Illustration of deriving higher order signatures from a signature containing at least a double transmission (TT)

One other interesting features of ray signature which is illustrated in Figure 8, is that it is possible to derive higher 
order signatures from a lower order signature. The order of a signature being defined as the number of interactions $N_{i}$ involved in the signature. For example if we have a signature of order $4: \mathcal{S}_{0}=\{1,2,3,4\}$ corresponding for example to the transmission through of 4 successive walls placed between $\mathbf{T}_{x}$ and $\mathbf{R}_{x}$, one can derive those 3 new signatures of order $6, \mathcal{S}_{1}=\{1,2,1,2,3,4\}, \mathcal{S}_{2}=\{1,2,3,2,3,4\}$, $\mathcal{S}_{3}=\{1,2,3,4,3,4\}$

\section{Relating Ray Persistency to Ray Signature}

Figure 9 presents the synthetic trajectory selected on the available measured link of an IR-UWB measurement campaign realized in the FP7-WHERE project [5]. Each trans-

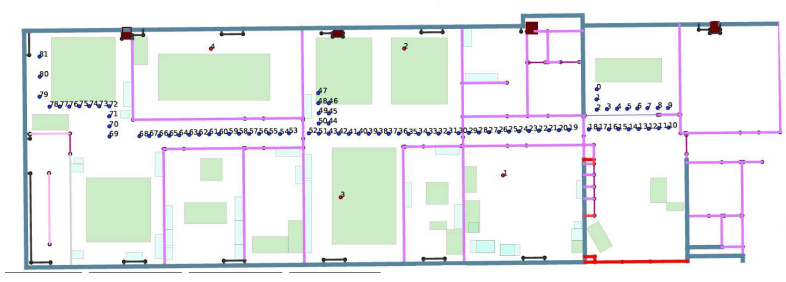

Fig. 9. Measured CIR along indoor selected trajectory, (red points) 4 Tx (1 is bottom right), (blue points) $81 \mathrm{Rx}$ ( 0 right -81 right)

mitter position $T x_{k}$ along the trajectory is numbered from $k=0$ to $k=81$. The trajectory starts on the right and the node moves from right to left. 4 Receivers are placed in the environment ( $R x_{1}$ bottom right to $R x_{4}$ up left). In figure 10, the Channel Impulse Response (CIR) of 4 links $\left\{T x_{k} R x_{1}, T x_{k} R x_{2}, T x_{k} R x_{3}, T x_{k} R x_{4}\right\}$ are shown. The horizontal axis is the delay expressed in nanoseconds of the CIR and the vertical axis corresponds to the synthetic trajectory index. Figure 11 is the noise free simulated CIR along the same trajectory with the ray tracing tool exploiting signatures. One can observe that there exist diagonal NLOS paths, meaning spatially coherent NLOS contributors which are often correctly predicted through RT simulation. An other interesting aspect is when one considers room transitions (e.g $R x 3 T x 53$ ) which are critical events regarding the CIR structure.

\section{CONCLUSION}

This paper has presented a technique for fast retrieving the ray signature from a graph description of the indoor layout. This signature is a precursor of rays and can be exploited to save computational time when evaluating the channel impulse response for example when studying indoor pedestrian localization or tracking scenario. In the last section ray persistency has been well observed in measurements data and well retrieved using an incremental ray tracing tool. A current line of investigation in tracking applications is to consider how to identify a ray signature from a sequential accumulation of channel impulse responses. This should be helpful in the context of map aided positioning and tracking scenarios.
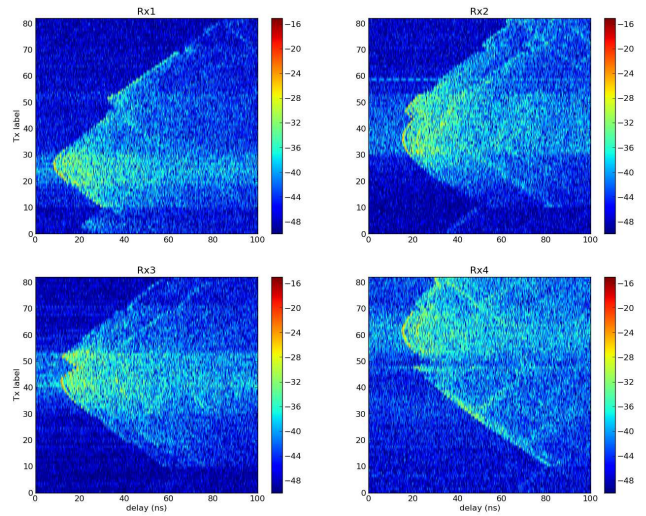

Fig. 10. Measured CIR along an indoor selected trajectory
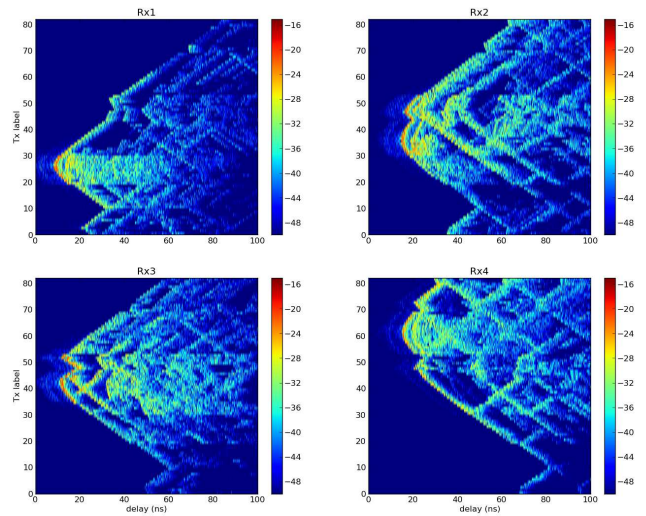

Fig. 11. Ray tracing simulated CIR along an indoor selected trajectory

\section{ACKNOWLEDGMENT}

The work has been performed in the FP7 project ICT248894 WHERE2, which is funded by the European Union

\section{REFERENCES}

[1] C.-C. Chong, C.-M. Tan, D. Laurenson, S. McLaughlin, M. Beach, and A. Nix, "A novel wideband dynamic directional indoor channel model based on a markov process," Wireless Communications, IEEE Transactions on 4 no. 4, (July, 2005) 1539 - 1552.

[2] J. Youssef, B. Denis, C. Godin, and S. Lesecq, "Dynamic ir-uwb channel model preserving ieee 802.15.4a statistics over large-scale trajectories," Personal, Indoor and Mobile Radio Communications, 2009 IEEE 20th International Symposium on (Sept., 2009) 1737-1741.

[3] F. Akgul and K. Pahlavan, "Path persistency for high precision ranging in different building architectures," in Personal, Indoor and Mobile Radio Communications, 2007. PIMRC 2007. IEEE 18th International Symposium on, pp. 1 -5. Sept., 2007.

[4] T. Pedersen and B. H. Fleury, "Radio channel modelling using stochastic propagation graphs," in Communications, 2007. ICC '07. IEEE International Conference on, pp. 2733-2738. 24-28 june, 2007.

[5] ICT-217033-WHERE-Project, "Deliverable 4.1: Measurements of location-dependent channel features,". 